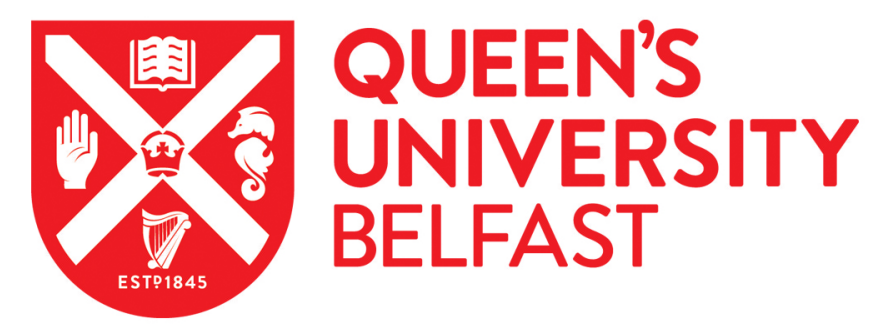

\title{
Performance Investigation of NOMA versus OMA Techniques for mmWave Massive MIMO Communications
}

Ghosh, J., Sharma, V., Haci, H., Singh, S., \& Ra, I-H. (2021). Performance Investigation of NOMA versus OMA Techniques for mmWave Massive MIMO Communications. IEEE Access. https://doi.org/10.1109/ACCESS.2021.3102301

\section{Published in: \\ IEEE Access}

\section{Document Version:}

Publisher's PDF, also known as Version of record

\section{Queen's University Belfast - Research Portal:}

Link to publication record in Queen's University Belfast Research Portal

\section{Publisher rights}

Copyright 2021 the authors.

This is an open access article published under a Creative Commons Attribution License (https://creativecommons.org/licenses/by/4.0/), which permits unrestricted use, distribution and reproduction in any medium, provided the author and source are cited.

\section{General rights}

Copyright for the publications made accessible via the Queen's University Belfast Research Portal is retained by the author(s) and / or other copyright owners and it is a condition of accessing these publications that users recognise and abide by the legal requirements associated with these rights.

Take down policy

The Research Portal is Queen's institutional repository that provides access to Queen's research output. Every effort has been made to ensure that content in the Research Portal does not infringe any person's rights, or applicable UK laws. If you discover content in the Research Portal that you believe breaches copyright or violates any law, please contact openaccess@qub.ac.uk. 


\title{
Performance Investigation of NOMA versus OMA Techniques for mmWave Massive MIMO Communications
}

\author{
JOYDEV GHOSH ${ }^{1}$, (Member, IEEE), VISHAL SHARMA ${ }^{2}$,(Member, IEEE), HÜSEYIN HACI ${ }^{3}$, \\ SAURABH SINGH ${ }^{4}$, (Member, IEEE), IN-HO RA ${ }^{5}$, (Member, IEEE) \\ ${ }^{1}$ School of Computer Science and Robotics, Tomsk Polytechnic University, Tomsk, Russia. (e-mail: joydev.ghosh.ece@gmail.com) \\ ${ }^{2}$ School of Electronics, Electrical Engineering and Computer Science, Queen's University Belfast, UK. (e-mail: vishal_sharma2012@hotmail.com) \\ ${ }^{3}$ Department of Electrical and Electronics Engineering, Near East University, Mersin 10, Turkey.(e-mail:huseyin.haci@neu.edu.tr) \\ ${ }^{4}$ Department of Industrial Systems Engineering, Dongguk University, South Korea.(e-mail:saurabh89@dongguk.edu) \\ ${ }^{5}$ School of Computer, Information and Communication Engineering, Kunsan National University, Gunsan 54150, Korea.(e-mail:Ihra@kunsan.ac.kr) \\ Corresponding author: S. Singh (e-mail:saurabh89@dongguk.edu), In-Ho Ra (e-mail:Ihra@kunsan.ac.kr)
}

It was supported by KETEP, Korean Government, Ministry of Trade, Industry, and Energy (MOTIE), grant number 20194010201800, and the National Research Foundation of Korea (NRF) grant funded by the Korea government (MSIT), grant number $2021 \mathrm{R} 1 \mathrm{~A} 2 \mathrm{C} 2014333$.

\begin{abstract}
The fifth-generation (5G) of cellular technology is currently being deployed over the world. In the next decade of mobile networks, beyond 5G (B5G) cellular networks with the under-development advanced technology enablers are expected to be a fully developed system that could offer tremendous opportunities for both enterprises and society at large. B5G in more ambitious scenarios will be capable to facilitate much-improved performance with the significant upgrade of the key parameters such as massive connectivity, ultra-reliable and low latency (URLL), spectral efficiency (SE) and energy efficiency (EE). Equipping non-orthogonal multiple access (NOMA) with other key drivers will help to explore systems' applicability to cover a wide variety of applications to forge a path for future networks. NOMA empowers the networks with seamless connectivity and can provide a secure transmission strategy for the industrial internet of things (IIoT) anywhere and anytime. Despite being a promising candidate for B5G networks a comprehensive study that covers operating principles, fundamental features and technological feasibility of NOMA at mmWave massive MIMO communications is not available. To address this, a simulation-based comparative study between NOMA and orthogonal multiple access (OMA) techniques for mmWave massive multiple-input and multiple-output (MIMO) communications is presented with performance discussions and identifying technology gaps. Throughout the paper, aspects of operating principles, fundamental features and technological feasibility of NOMA are discussed. Also, it is demonstrated that NOMA not only has good adaptability but also can outperform other OMA techniques for mmWave massive MIMO communications. Some foreseeable challenges and future directions on applying NOMA to B5G networks are also provided.
\end{abstract}

INDEX TERMS NOMA, OMA, Massive MIMO, mmWave Communication, 3GPP, PAPR

\section{INTRODUCTION}

As of today fourth generation $(4 \mathrm{G})$-cellular networks have already been launched worldwide. However, $4 \mathrm{G}$ is not well capable to provide ubiquitous connectivity and all other demands of both individual consumers and businesses, while fifth-generation $(5 \mathrm{G})$ has transformed into an enhanced mobile broadband (eMBB) with the Internet of Things (IoT) from MBB of the existing 4G. The IoT further incorporates $5 \mathrm{G}$ use-cases (massive machine-type communications (mMTC), ultra-reliable and low latency communication (URLLC)) and market verticals. The physical layer design for URLLC in the third generation partnership project
(3GPP) Release 15 new radio (NR) was announced at the end of 2017 [1]. Compared to orthogonal multiple access (OMA) used in 4G, orthogonal frequency division multiplexing (OFDM) of $5 \mathrm{G}$ can provide faster transmission speed by reducing the time duration of OFDM symbols and cyclic prefix (CP) length with the help of dynamic sub-carrier spacing (SCS) [2]. In future we may offer an in-home IoT remote health monitoring system by linking medical devices with URLLC, this can only be successful in $5 \mathrm{G}$ and beyond (5GB) networks with the inclusion of nonorthogonal multiple access (NOMA), massive multi-input multi-output (mMIMO) and millimetre wave (mmWave) as 
the enabling technologies [3-10]. Since only then the massive connectivity and related ultra high traffic can be handled by the communication networks [11].

In [12], the authors introduced cognitive wireless powered communication networks (CWPCNs) which operate on the concept of a harvest-then-transmit (HTT) protocol. In this full-duplex (FD) communication strategy, users initially performed energy harvesting (EV) during downlink (DL) transmission of an access point (AP) and thereafter using that energy data transmission took place at AP via relay assistance by applying either time division multiple access (TDMA), orthogonal frequency division multiple access (OFDMA) or NOMA [13],[14]. Although NOMA-driven CWPCN is a better choice due to its numerous advantages over the other two schemes, NOMA technology has not been widely deployed in real-life applications yet. These days due to massive IoT (mIoT) deployment, NOMA is now becoming a more favourable and feasible multiple access (MA) technique than conventional OMA. An energy-efficient simultaneous wireless information and power transfer (SWIPT)-assisted NOMA system was presented in [15-18] and [19] in order to deal with spectrum sensing overhead and battery draining issues of cognitive radio (CR) network, and to enable massive connectivity at underutilized spectrum. [20],[21] applied NOMA for the massive connectivity and explored energy efficiency (EE) of mobile edge computing (MEC) in IoT networks. The application of NOMA is also valid in vehicular edge computing networks (VECNs), which integrate MEC and vehicular networks. The authors in [22], [23] studied the advantages of NOMA assisted beamspace MIMO over conventional-beamspace MIMO, it has been shown that the restriction on the limited number of radio frequency (RF) chains had been significantly improved. [2427] proposed a novel hybrid transceiver design for MIMONOMA deploying lens antenna array. In [28-34], simulation results demonstrated that compared to OMA, NOMA has better performances in terms of channel estimation accuracy, system sum-rate, outage probability and symbol error rate (SER). [35] shown that the installation of reconfigurable intelligent surface (RIS) in MIMO-NOMA for long-distance communication could be an effective solution to the EE optimization problem using its reconfigurable properties combined with the higher degrees of freedom provided with the frequency reuse capability of NOMA. Moreover, a novel power consumption model with varactor diodes designed for NOMA communications had also been presented. In [36] it has been shown that NOMA-based unmanned aerial vehicle (UAV) network compared to OMA-based UAV network could be a better choice concerning several performance metrics, such as EE and spectral efficiency (SE). [37] provided the analysis and techniques of EE optimization for NOMA-UAV networks subjected to imperfect channel state information (ICSI) and interference. According to [38], the wireless IoT network design with solar-powered UAV and NOMA could be a very useful tool to building a sustainable energy future where NOMA-based design aids with the joint problem of altitude control and stochastic control. UAV-Base Stations (UAV-BSs) can serve multiple terminals over the same resource block with the help of integrating NOMA, hence it can effectively improve the SE. In [39-41], under the NOMA context, authors used hybrid precoding (HP) in mmWave massive MIMO-NOMA for SWIPT that in turn helped to reduce RF chains and maximized EE. The releases of 3GPP R1-163887 [42] / R1-165484 [43] had given in detail time-varying properties of mmWave high-speed train (HST) channel on latency and Doppler shift which could be interesting foundations to develop NOMA-based system at highly mobile networks. Integrating NOMA with MTC had also recognized by $3 \mathrm{GPP}$ release 11 in the advancement of cellular systems. The authors in [44] presented 3GPP licensed assisted access (LAA) with its configurable radio operation scenarios with NOMA. The article had also shown LAA's feasibility with small cells, particularly femtocells, and associated challenges for seamless wireless services and mobility management.

The present 3GPP specification recognizes accomplishment for only wireless local area network (WLAN) as the untrusted non-3GPP access network (AN). A user needs to be connected with the Non-3GPP interworking function (N3IWF) to have access to the $5 \mathrm{G}$ core network (5GCN) via a non-3GPP AN. The non-3GPP AN has brought much attention even today due to its application in public hotspots and home/corporate Wi-Fi connectivity. Based on a recent release, non-3GPP AN can complement 3GPP access networks in a single $5 \mathrm{GCN}$ by assigning different internet protocol (IP)-based services, which helps to reduce data congestion, much-improved coverage and connectivity in high traffic volume scenarios, less operational costs and open-up new business opportunities. NOMA enabled AN technologies can be a good candidate for non-3GPP AN at 5GCN.

Despite interesting researches mentioned above, a comprehensive performance comparison of NOMA and OMA based mmWave Massive MIMO communications has not been studied yet. To address this, in this article we present an up-to-date overview of the MIMO-NOMA scheme for the envisioned B5G wireless communication to spark an interest in the results and reports of recent studies, including touch on the partial development of NOMA in 3GPP. We try to provide a comprehensive idea of the interplay between MIMONOMA and other OMA schemes by adopting SE, EE and outage probability $(\mathrm{OP})$ as performance metrics. This leads us to produce the simulation results on two research thrust areas such as international telecommunication union (ITU) channel models and peak-to-average-power ratio (PAPR) for the NOMA scheme in this article for the first time.

\section{PERFORMANCE OF NOMA OVER DIFFERENT MIMO MMWAVE SCHEMES}

NOMA is regarded to be one of quite a few technologies which can enhance the overall capacity of the $5 \mathrm{G}$ network. NOMA involves applying superposition coding (SC) at the transmitter and successive interference cancellation (SIC) at 
Table 1: System model and advantages/disadvantages of different schemes

\begin{tabular}{|c|c|c|}
\hline Scheme & Fundamental technologies & Advantages/Disadvantages \\
\hline \multirow[t]{5}{*}{ Perfectly Digital MIMO Network } & 1. Perfectly digital precoder & Increases number of RF chains \\
\hline & 2. Deployment of RF chain to each antenna element & Challenging due to per antenna non-linearity \\
\hline & 3. Analog-to-digital converter (ADC) & Increases power loss and hardware cost \\
\hline & 4. Silicon realizations of mmWave hardware & $\begin{array}{l}\text { Makes this feasible to accommodate a large number of } \\
\text { RF chains }\end{array}$ \\
\hline & 5. Fully digital spatial processing & $\begin{array}{l}\text { Helps to accommodate a large number of users simulta- } \\
\text { neously }\end{array}$ \\
\hline \multirow[t]{6}{*}{ Beamspace MIMO Network } & $\begin{array}{l}\text { 1. Continuous aperture phased (CAP)-MIMO transceiver } \\
\text { architecture }\end{array}$ & Reduces the transceiver complexity \\
\hline & 2. An interference-aware (IA) beam selection scheme & Provides safety from multiuser interferences \\
\hline & 3. Discrete lens array (DLA)-based prototype & $\begin{array}{l}\text { Creates favourable condition for line-of-sight }(\operatorname{LoS}) \text { com- } \\
\text { munication }\end{array}$ \\
\hline & $\begin{array}{l}\text { 4. Joint operation of beamspace-MIMO precoders and } \\
\text { analog beamforming }\end{array}$ & $\begin{array}{l}\text { Provides perfect balance between complexity and perfor- } \\
\text { mance in mmWave scenarios }\end{array}$ \\
\hline & 5. Orthogonal spatial beams & $\begin{array}{l}\text { Helps to perform well at the lower signal to noise ratio } \\
\text { (SNR) }\end{array}$ \\
\hline & 6. Beamspace channel sparsity & Provides better channel estimation quality \\
\hline \multirow[t]{4}{*}{ MIMO OMA mmWave Network } & 1. A multi-user version of OFDM modulation & Higher sensitive to frequency selective fading \\
\hline & 2. Hybrid multi-user equalizer & Reduces hardware cost and energy consumption \\
\hline & $\begin{array}{l}\text { 3. Constant envelope (CE)-OFDM modulation in } \\
\text { mmWave }\end{array}$ & Increases number of subcarriers and maintain low PAPR \\
\hline & 4. Flexible $\mathrm{CP}$ & Reduces throughput, but helps to achieve low latency \\
\hline \multirow[t]{4}{*}{ MIMO NOMA mmWave Network } & 1. Superposition coding & $\begin{array}{l}\text { Each data stream can concurrently support multiple UEs } \\
\text { at the same time-frequency resource block }\end{array}$ \\
\hline & 2. SIC receiver & Helps to combat multiple-access interference \\
\hline & 3. Large phased arrays & Helps to compensate high propagation loss \\
\hline & 4. Multi-beam forming & Improves sum-rate performance \\
\hline
\end{tabular}

the receiver to obtain the capacity area in the DL broadcast channel, which in turn outperforms OMA. Mainly, NOMA has been developed to optimize the network's SE, with slight focus taking into account the EE.

In this section, we illustrate simulation outcomes for validating the performance of mmWave MIMO-NOMA network in DL transmission link, where the BS is supplied with 256 antennas and connects with 45 user equipments (UEs). A single line-of-sight (LoS) component and two non-line-of-sight $(\mathrm{NLoS})$ components are incorporated for all UE channels. We consider the following assumption for the channel estimation of $x^{\text {th }} \mathrm{UE}$ [45]: (i) $\zeta_{x}^{o} \sim \mathcal{C N}(0,1), \zeta_{x}^{l} \sim \mathcal{C N}\left(0,10^{-1}\right)$ for $0 \leq l \leq L$ where $\zeta_{x}^{o}$ and $\zeta_{x}^{l}$ stand for complex gain and $\mathcal{C} \mathcal{N}(a, b)$ represents complex normal distribution with mean $a$ and variance $b, L$ denotes total number of NLoS components and $l$ is used to denote NLOS component associated to $x^{\text {th }}$ user; (ii) spatial direction is denoted by $\phi_{x}^{o}$ and $\phi_{x}^{l}$ for $0 \leq l \leq L$ comply with the independent and identically distributed (i.i.d) unvarying distribution over $\left[-\frac{1}{2}, \frac{1}{2}\right]$.
We compare the following four typical MIMO mmWave techniques: 1) 'Perfectly Digital MIMO Network' [46], [47], where the individual antenna is linked to an RF chain, 2) 'Beamspace MIMO Network' [48], [49], where individual beam facilitates a UE 3) 'MIMO OMA mmWave Network' [50], where OMA is executed for the interfering UEs, and UEs located in the alike beam is assigned with orthogonal spectrum resources; 4) 'MIMO NOMA mmWave Network' [45], which harmonizes NOMA into Beamspace MIMO, and different UEs utilize the alike time and spectrum resources. Besides, zero-forcing (ZF) pre-coding is added in the perfectly digital MIMO network and beamspace MIMO network. A summary of the fundamental technologies of each scheme and associated advantages/disadvantages are provided in Table 1.

\section{A. IMPROVED SE}

Figure 1 depicts SE comparison of NOMA mmWave scheme over other mmWave schemes, where an essential iteration 


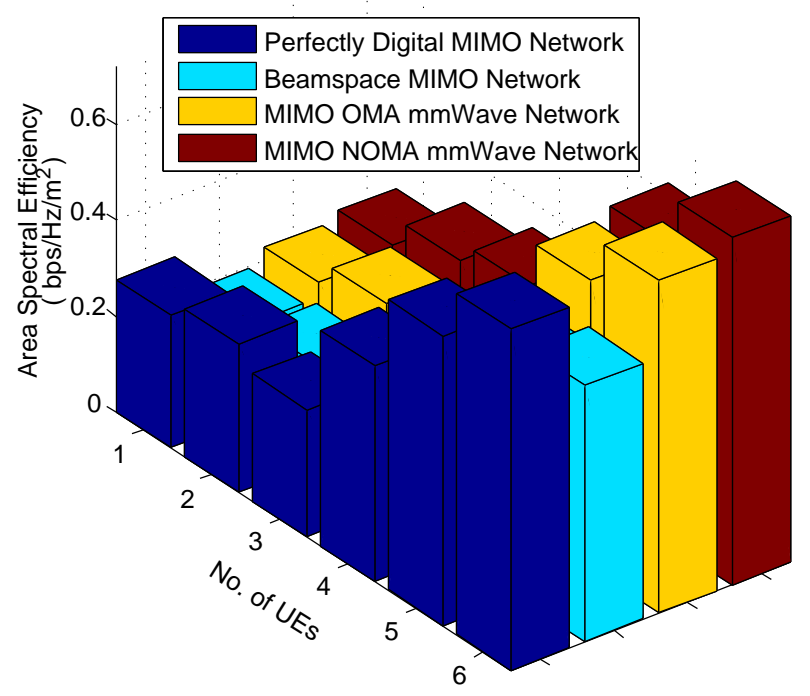

Figure 1:SE comparison of different channel multiple accessbased MIMO mmWave techniques

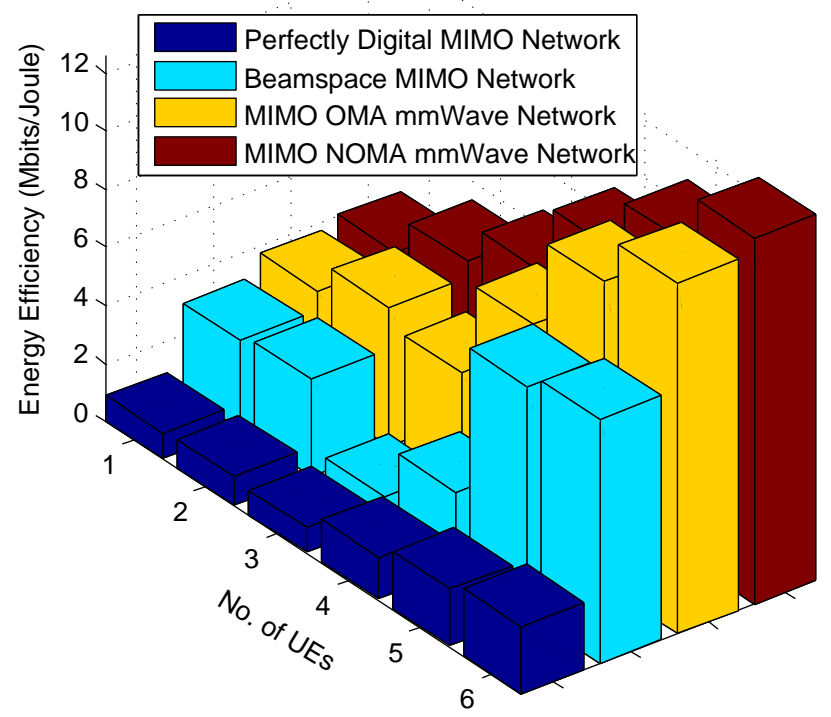

Figure 2: EE comparison of different channel multiple access-based MIMO mmWave techniques

number and iteration time to resolve the power assignment optimization difficulty is set as 15 , at which SE becomes a constant, and 30, which is enough to converge the power assignment algorithm, respectively. We reveal that MIMONOMA mmWave network obtains a larger SE compare to that of Beamspace MIMO Network and MIMO-OMA mmWave network. Concerning quantity, MIMO-NOMA mmWave network has almost around $5 \mathrm{~dB}$ SNR gain (as the average total SNR of users accessing same carriers simultaneously) than the MIMO-OMA mmWave network (a single user accessing a carrier at a time), which is beneficial from the point of view of NOMA deployment to facilitate more
UEs in each beam.

This can also be noticed that a perfectly digital MIMO network can obtain the highest level of SE over other mmWave schemes, as beam selection is no way linked to a perfectly digital MIMO network, and an applied number of BS antennas as RF chains are considered to facilitate all UEs. Although, a perfectly digital MIMO network becomes worse in EE performance as illustrated in Figure 2.

\section{B. IMPROVED EE}

A comparison of four schemes in terms of $\mathrm{EE}$ as a function of SNR is demonstrated in Figure 2, where the number of UEs is set at 45. Here, again we observe that MIMONOMA mmWave network outperforms the rest schemes in terms of EE. Precisely, MIMO-NOMA mmWave network has almost around $15 \%$ improvement in EE performance than the MIMO-OMA mmWave network, which is beneficial from the point of view of NOMA deployment to facilitate more UEs in each beam.

Besides, EE of the MIMO-NOMA mmWave network is more than a perfectly digital MIMO network, where RF chains equal to applied antennas by its number which results in extremely high energy utilization, e.g., $200 \mathrm{~mW}$ for each RF chain. In contrast to a perfectly digital MIMO network, RF chains in number are even lesser than the applied antennas to a great extend in MIMO-NOMA mmWave network. Hence, the energy utilized by the RF chains can be remarkably decreased in NOMA than perfectly digital MIMO network.

Likewise, it can also be seen that MIMO-NOMA mmWave network outperforms the other schemes on SE and EE for the increasing number of UEs. It is because the higher number of UEs leads to the high possibility that the same beam can be chosen for different UEs, which in turn deteriorate the performance of the beam space MIMO network. However, the performance level of the MIMO-NOMA mmWave network cannot be affected due to the application of NOMA technology which can accommodate the higher number of UEs than OMA by applying non-orthogonal resource allocation.

\section{3GPP CASE STUDIES}

In $5 \mathrm{G}$ and $\mathrm{B} 5 \mathrm{G}$ networks it is expected that major share of the data traffic will be of interactive type (e.g. real-time video and Voice over IP) where both DL and uplink (UL) transmissions' performance play equal share to enable high quality of service (QoS) communications. Another communication's aspect that has not been studied enough is the performance of mmWave massive MIMO communications under various mobility models. Therefore in this section, performance comparison of MIMO-NOMA and MIMO-OMA for DL and UL transmissions are provided under four different channel models, such as Rayleigh fading channel and three 3GPP/ITU channel models that model different mobility profiles as Pedestrian A-channel, Pedestrian B-channel and Vehicular A-channel. The EE to SE trade-off performance for MIMONOMA and MIMO-OMA has been shown in Figs. 3 and 


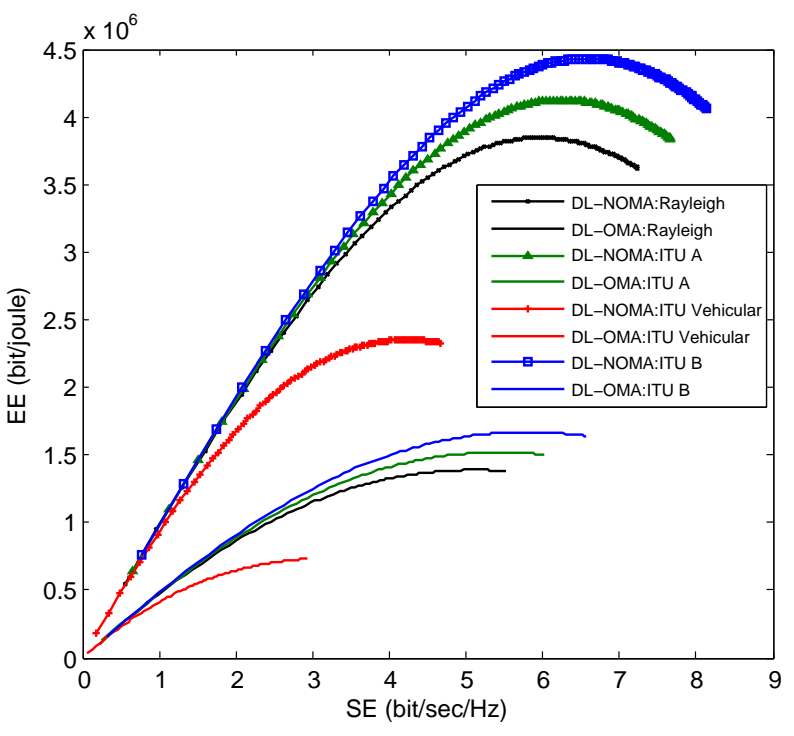

Figure 3:3GPP case studies for EE to SE trade-off for DL transmissions

4 for DL and UL transmissions, respectively. It can be seen from the figures that MIMO-NOMA can outperform MIMO-OMA for all the considered channel models and SE at DL and UL transmissions. EE at the DL transmissions is important to reduce the carbon footprint of the AN, and UL transmissions to prolong the UE's battery life as well as the carbon footprint. Another important observation is that the performance gap increases as the SE increases. Thus, in future ultra-high-speed communications, MIMO-NOMA can provide multiple times better EE to SE performance than MIMO-OMA. Further, performance comparison concerning increasing mobility profile is an interesting aspect for $5 \mathrm{G}$ and $\mathrm{B} 5 \mathrm{G}$ communications. It can be seen from the figures that as the mobility profile increases, i.e. from Rayleigh (no mobility), ITU Pedestrian A, ITU Pedestrian B to ITU Vehicular A, the performance gap increases as well. This indicates that MIMO-NOMA is a favourable scheme over MIMO-OMA schemes for all range of mobility profiles and especially at high mobility scenarios.

Despite the performance superiority of NOMA over OMA, NOMA should be deployed with precautions towards security and privacy. NOMA has the special features of massive connectivity and multiple users can share the same resource block, which may make NOMA more vulnerable to various malicious attacks. 3GPP and non-3GPP B5G networks may face security issues at different operation stages, such as radio link establishment, data exchange and link reconfiguration stages. Thus security procedures need to be incorporated at each of these stages. [51] studied physical layer security for NOMA with limitations and possible recommendations for solutions. As NOMA can be adopted by many cuttingedge technologies, there is a high possibility of end-user privacy violation threats especially in the following popular

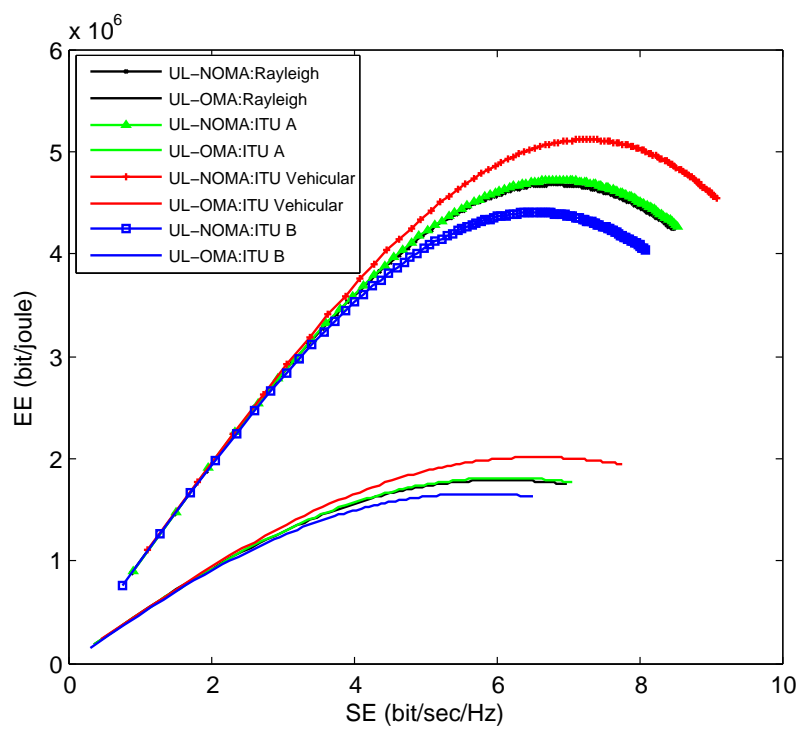

Figure 4: 3GPP case studies for EE to SE trade-off for UL transmissions

application areas that involve the exchange of end-users' private information, massive IoT device access, device-todevice (D2D) and vehicle to everything (V2X) communications. Security procedures to be applied in these areas should be chosen not to violate end-users' privacy, as discussed in [52].

\section{PEAK TO AVERAGE POWER RATIO (PAPR) MANAGEMENT}

The basic concept of OFDM can be described by the concealed fundamental pulses. Precoding by the spreading matrix changes the fundamental pulses into new estimated fundamental pulses. The optimum dimension of the spreading matrix and hence the number of new estimated fundamental pulses is determined by the precoding technique. Figure 5 depicts the fundamental pulses' power versus frequency spectrum and demonstrates a step by step building of various popular schemes, beginning from a conventional OFDM technique. The OFDM [53] in Figure 5 is illustrated for Fast Fourier Transform (FFT) size equals 512, subcarrier size equals 16 and OFDM symbol equals 1 . The concealed fundamental pulses are denoted or shaped like a rectangle and are a function of frequency displacement. Entire fundamental pulses are appended together with some haphazard weights and are represented by the data symbols, thus the information dispersal in a sample invokes a normal distribution-based on the central limit theorem (CLT). This results in a high PAPR problem in OFDM. In Single Carrier Frequency Division Multiple Access (SC-FDMA) [54] precoding by discrete Fourier transform (DFT), matrix changes the fundamental pulses of an OFDM technique into a single carrier transmission. Precisely, the fundamental pulses are confined in time to a great extent and even though they overlap the information at a sample is usually resolute from few fundamental pulses. 


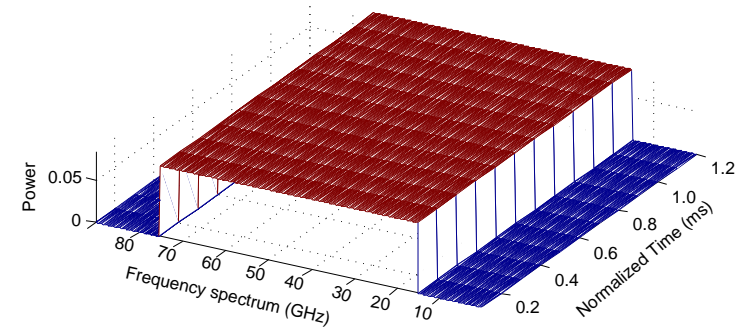

OFDM

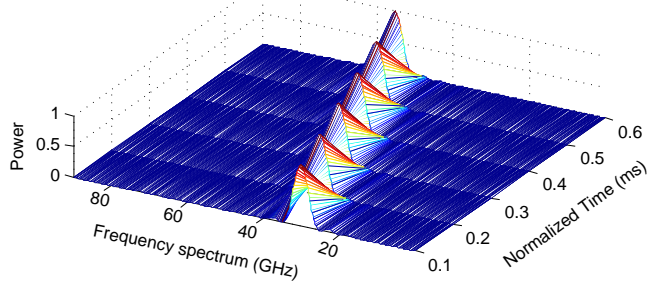

Zero-tail OFDM

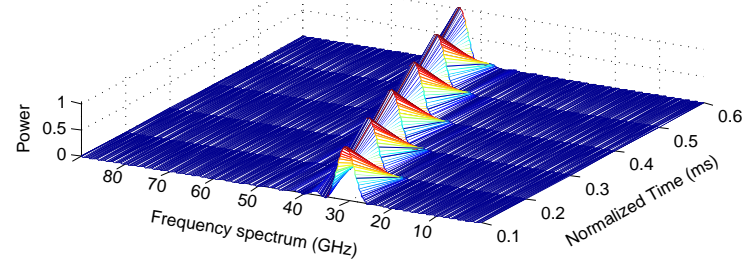

Zero-tail Pruned FBMC

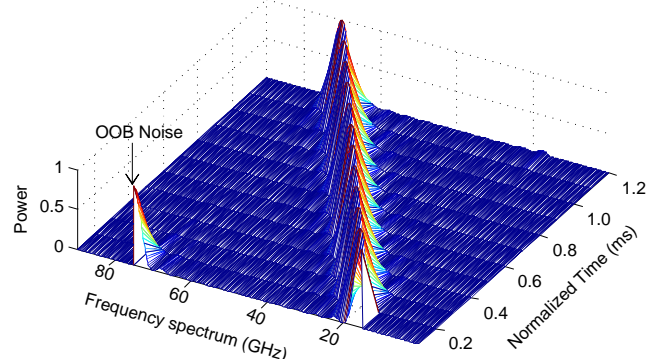

SC-FDMA

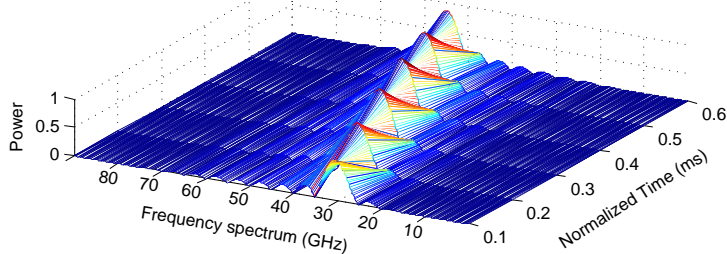

Pruned FBMC

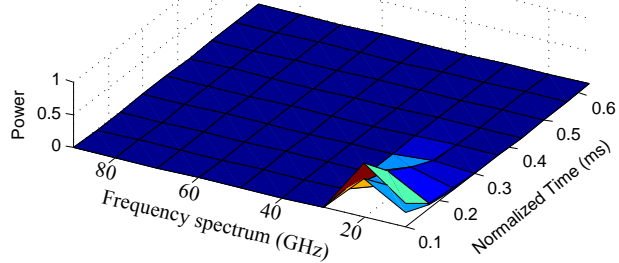

Precoded NOMA

Figure 5: Fundamental pulses power response of NOMA and different OMA techniques

Therefore, PAPR responses are considerably better compared to that of OFDM in case data symbols can be represented by a normal distribution. However, SC-FDMA suffers from the out of band (OOB) noise. That is resulted by incorporating the communicated signal at the edge locations, i.e., normalize time equals 0 and 1 . The concealed fundamental pulse cuts through the signal at the edges like OFDM, as a result, the signal unexpectedly shift to zero and do not follow a flat transition. The fundamental pulses near the edge locations can be suppressed with the clipping effect. Hence, adjusting the edge fundamental pulses to zero attenuates the OOB noise as illustrated by the fundamental idea of the zero-tail OFDM scheme in Figure 5. Some of the fundamental pulses can be eliminated to keep the zero-tail overhead low. To tackle delay spread, which is a metric to measure the multipath severity of a communications channel, the zero-tail overhead in zero-tail OFDM applies similarly to the CP in OFDM. This results in deterioration of performance in terms of SE. Therefore, another technique has been considered and hence an OFDM technique is transformed into an FBMC technique to mitigate interference to a negligible amount. The transformation of an OFDM technique into an unscaled FBMC technique can be achieved from the product of the inverse fast fourier transform (IFFT) outcome with a prototype filter, as shown in Figure 5. Each of the fundamental pulses apart from the zero-tail Pruned FBMC technique is maximizing in order to make almost constant power during the transmission.

FBMC technology finds its applications in the following areas, but not limited to: CR Communications, multiple access networks, access to television white space (TVWS), power line communication (PLC), MIMO Communication. FBMC is beneficial due to the following reasons: outperforms OFDMA in terms of both Bit error rate (BER) and PAPR, utilizes best quality filters that mitigate both ingress and egress noises, the removal of the sidelobes spread out either side applying the filter bank system results in a much cleaner carrier outputs, less sensitive to carrier frequency offset (CFO), higher bandwidth efficiency, higher spectrum sensing resolution, supports asynchronous sub-bands. But it suffers from many shortcomings such as: lesser MIMO compatibility, data flow is considered to be unidirectional and data rates are considered to be constant throughout the 


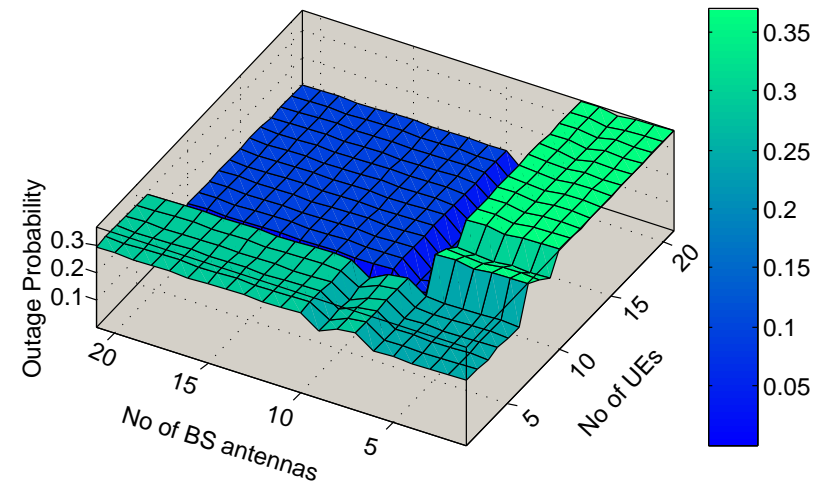

Figure 6:Performance comparison of outage probability for MIMO-NOMA

operation of the network without assuming any feedback operations, a uniform power delay profile is taken into account, no interferences from external sources are assumed, increase the training overhead for overlapping symbols in the filter bank over the time domain.

The High PAPR issue is one of the major shortcomings in the non-precoding NOMA scheme. Hence the reduction of PAPR can make NOMA an efficient multiple access technologies. This problem can be resolved by adding a precoderbased on a discrete-cosine transform matrix (DCTM). The DCTM precoder reduces the autocorrelation state of being connected to the regulated data and propagates the information over individual subcarrier uniformly. To achieve little autocorrelation by taking the IFFT input, precoded regulated symbols may produce small in-phase insertion. Therefore, PAPR is decreased. The information propagation by the DCTM precoder grasps the benefits of multipath induced fading channel and decreases the symbol-error-rate (SER) to a great extent. The precoding out-turn can be described by creating an IFFT of size 300. The precoded succession generated due to the DCTM precoder has small side-lobe magnitudes in contrast to the side-lobe magnitudes of nonprecoded succession. Small side-lobe magnitudes indicate autocorrelation in small-scale, whereas large side-lobe magnitudes refer to large autocorrelation. Small-scale autocorrelation in IFFT input minimizes the probability of in-phase insertions at IFFT. As a result, PAPR is minimized. Thus, the conclusion can be drawn that a significant PAPR reduction is possible for the application of precoding to the constellation symbols before the IFFT operation.

\section{A. OUTAGE PERFORMANCE OF MIMO-NOMA NETWORK OVER CONVENTIONAL MIMO-OMA NETWORK}

In this section, the DL communication framework of a MIMO-NOMA network with the deployment of a single BS and various UEs has been discussed. This scenario is in association with massive IoT deployments where the number of UEs is much more compared to the available BSs. In

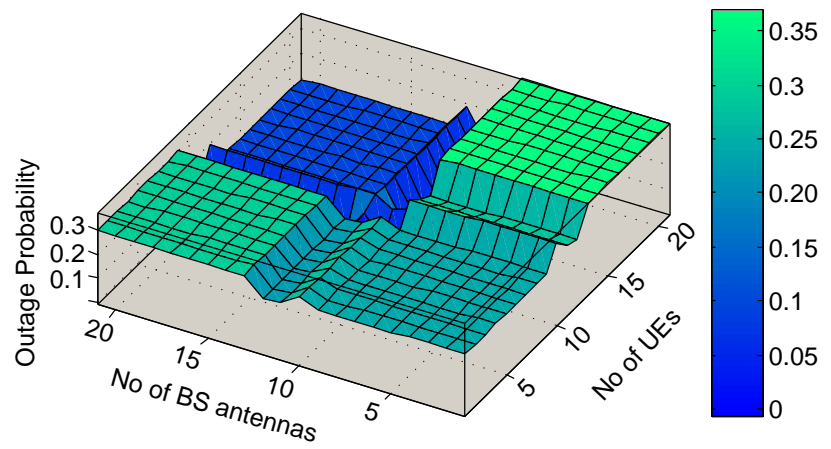

Figure 7:Performance comparison of outage probability for MIMO-OMA schemes

such a scenario each BS need to have a large number of antennas to be able to fulfil the performance requirements of IoT applications. Here, a UE (called a primary UE) is served by allocating enough radio resources to fulfilling its QoS requirements, whereas, the rest of the UEs occupy the same radio resources opportunistically by applying the NOMA technology. It should be noted that NOMA users access the same radio resources in a way not to degrade the primary UEs QoS below acceptable tolerance. Assume $M$ and $N$ antennas are used for equipping the BS and a UE, respectively. To realize NOMA's potential, two network metrics, namely precoding matrix and power allotment coefficient are carefully considered to fulfil the QoS requirements. Each element in the precoding matrix at the BS are i.i.d. complex Gaussian variable with zero mean and unit variance. The work presented in this section is articulated on the network scenario without path loss. To ensure the fast transmission of small data packets in a certain data rate for an IoT device, the BS transmits the signal in the form of the following vector: $v=C \bar{x}$ where $C$ stands for $M \times N$ channel matrix and $\bar{x}$ denotes a vector which carries the information. Thus, $\bar{x}$ can be formulated applying NOMA as follows [55]: $\bar{x}=\left[\alpha_{1} x_{1}+\cdots+\alpha_{M} x_{k}\right]^{T}$ where $\alpha_{i}$ indicates NOMA power allotment co-efficient and $x_{i}$ is the $i^{t h}$ small packet transmitted to a UE.

In Figs. 6 and 7, the outage probability performance of MIMO-NOMA and MIMO-OMA schemes are demonstrated as a function of a various number of antennas at the BS and UEs served by the BS. The MIMO-NOMA-based network can provide significantly better outage probability performance compared to that of MIMO-OMA-based network, as shown in Figs. 6 and 7. It is well known that for realtime video and voice communications the acceptable outage probability should be no more than $1 \%$. It can be seen from the figure that the MIMO-NOMA system can accommodate a significantly more number of users compared to a MIMOOMA system with a given number of antennas at a BS. An interesting finding is that the shape of the surfaces of the outage probability plots for MIMO-NOMA is quite similar to that of MIMO-OMA, which signifies that a similar diversity 
gain is produced by both types of networks. This occurrence is anticipated as identical effective channel gain, which is considered to compute the outage probability for both types of networks.

\section{CONCLUSION}

In this article, we have discussed and compared NOMA technology with OMA for mmWave massive MIMO-based wireless networks from the aspects of operating principles, fundamental features and technological feasibility. NOMA technology has the drawbacks of increased complexity at the receiver and limited co-user interference due to nonorthogonal resource allocation. However, this paper has shown that if appropriate resource allocation and precoding can be applied to NOMA, mmWave massive MIMO-NOMAbased communications can provide significant improvement in SE, EE and outage probability performances compared to MIMO-OMA. Also, the high PAPR problem of OFDMbased MIMO-OMA systems can be improved.

\section{REFERENCES}

[1] T.K. Le, U. Salim, F. Kaltenberger, "An Overview of Physical Layer Design for Ultra-Reliable Low-Latency Communications in 3GPP Releases 15, 16, and 17," IEEE Access, pp.433 - 444, vol. 9, 2020.

[2] X. Zhao, W. Cheng, H. Zhu, C. Ge, G. Zhou, Z. Fu, "A High Gain, Noise Cancelling 2515-4900 MHz CMOS LNA for China Mobile 5G Communication Application," CMC-Computers, Materials and Continua, vol.64, no.2, 2020, pp.1139-1151, doi:10.32604/cmc.2020.010220.

[3] T. Jiang, J. Zhang, P. Tang, L. Tian, Y. Zheng, J. Dou, H. Asplund, L. Raschkowski, R. D’Errico, T. Jämsä, “3GPP Standardized 5G Channel Model for IIoT Scenarios: A Survey,’IEEE Internet of Things Journal, DOI: 10.1109/JIOT.2020.3048992.

[4] J. Yang, B. Ai, K. Guan, D. He, X. Lin, B. Hui, J. Kim, A. Hrovat, "A Geometry-Based Stochastic Channel Model for the Millimeter-Wave Band in a 3GPP High-Speed Train Scenario," IEEE Transactions on Vehicular Technology, vol. 67, No. 5, pp.3853 - 3865, May 2018.

[5] H. Haci, H. Zhu, J. Wang, "Performance of non-orthogonal multiple access with a novel asynchronous interference cancellation technique," IEEE Transactions on Communications, vol.65, no.3, pp.1319-1335, 2017.

[6] Z. Ding, P. Fan, and H. V. Poor, "Impact of device pairing on 5 G nonorthogonal multiple-access downlink transmissions," IEEE Trans. Veh. Technology, vol. 65, no. 8, pp. 6010-6023, Aug. 2016.

[7] J. Ghosh, H. Zhu, H. Haci,"A Novel Channel Model and Optimal Beam Tracking Schemes for Mobile Millimeter-Wave Massive MIMO Communications" IEEE Transactions on Vehicular Technology, DOI:10.1109/TVT.2021.3083635.

[8] B. Chen, S. Qiao, J. Zhao, D. Liu, X. Shi, M. Lyu, H. Chen, H. Lu, Y. Zhai, "A Security Awareness and Protection System for 5G Smart Healthcare Based on Zero-Trust Architecture," IEEE Internet of Things Journal, vol. 8, No. 13, pp.10248 - 10263, 2021.

[9] R. M. Christopher, D. K. Borah, "Physical Layer Security for Weak User in MISO NOMA Using Directional Modulation (NOMAD)," IEEE Communications Letters, vol. 24, no. 5, pp.956 - 960, 2020.

[10] H. Shi, C. Pan, L. Yang, D. Wei, Y. Shi, "End-to-End Latency Evaluation of the Sat5G Network Based on Stochastic Network Calculus," CMCComputers, Materials and Continua, vol.65, No.2, 2020, pp.1335-1348, doi:10.32604/cmc.2020.011005.

[11] D. Y. Kim, S. Kim, "Network-Aided Intelligent Traffic Steering in 5G Mobile Networks," CMC-Computers, Materials and Continua, vol.65, no.1, 2020, pp.243-261, doi:10.32604/cmc.2020.011253.

[12] L. Liu, R. Zhang, and K.-C. Chua, "Multi-antenna wireless powered communication with energy beamforming," IEEE Trans. Commun., vol. 62, no. 12, pp. 4349-4361, 2014.

[13] J. Li, D. Mei, D. Deng, I. Khan, P. Uthansakul, "Proportional FairnessBased Power Allocation Algorithm for Downlink NOMA 5G Wireless Networks,' CMC-Computers, Materials and Continua, vol.65, no.2, 2020, pp.1571-1590, doi:10.32604/cmc.2020.011822.
[14] Y. Zhang, J. Liu1, Y. Peng, Y. Dong, G. Sun, H. Huang, C. Zhao, "Performance Analysis of Relay Based NOMA Cooperative Transmission under Cognitive Radio Network," CMC-Computers, Materials and Continua, vol.63, no.1, 2020, pp.197-212, doi:10.32604/cmc.2020.07059.

[15] J. Tang, Y. Yu, M. Liu, D. K. C. So, X. Zhang, Z. Li, and K.-K. Wong, "Joint power allocation and splitting control for SWIPT-enabled NOMA systems," IEEE Trans. Wireless Commun., vol. 19, no. 1, pp. 120-133,Jan. 2020.

[16] X. Wang, Z. Na, K.Y. Lam, X. Liu, Z. Gao, F. Li, L. Wang, "Energy Efficiency Optimization for NOMA-Based Cognitive Radio With Energy Harvesting," IEEE Access, vol. 7, pp.139172 - 139180, 2019.

[17] H. Yang, X. Xia, J. Li, P. Zhu, Xiaohu You, “Joint Transceiver Design for Network-Assisted Full-Duplex Systems With SWIPT," IEEE Systems Journal 2021, DOI: 10.1109/JSYST.2021.3062455.

[18] M. Thirunavukkarasu, R. J. Sparjan, L. Thangavelu, "An Adaptive Power Allocation Scheme for Performance Improvement of Cooperative SWIPT NOMA Wireless Networks," CMC-Computers, Materials and Continua, vol.63, no.2, 2020, pp.1043-1064, doi:10.32604/cmc.2020.09819.

[19] J. Tang, J. Luo, M.Liu, D. So, E. Alsusa, G. Chen, K.K Wong, and J. Chambers, "Energy Efficiency Optimization for NOMA With SWIPT,' IEEE Journal of Selected Topics in Signal Processing, vol. 13, No. 3, pp.452 - 466, June 2019.

[20] B. Liu, C. Liu, and M. Peng, "Resource Allocation for Energy-Efficient MEC in NOMA-Enabled Massive IoT Networks," IEEE Journal on Selected Areas in Communications, vol. 39, No. 4, pp.1015 - 1027, April 2021.

[21] L. Shi, Y. Ye, X. Chu, G. Lu, “Computation Energy Efficiency Maximization for a NOMA-Based WPT-MEC Network," IEEE Internet of Things Journal, vol. 8, no. 13, pp.10731 - 10744, 2021.

[22] B. Wang, L. Dai, Z. Wang, N. Ge, and S. Zhou, "Spectrum and energy efficient beamspace MIMO-NOMA for millimeter-wave communications using lens antenna array," IEEE Journal on Selected Areas in Communications, vol. 35, no. 10, pp. 2370-2382, 2017.

[23] P. V. Amadori and C. Masouros, "Low RF-complexity millimeter-wave beamspace-MIMO systems by beam selection," IEEE Transactions on Communications, vol. 63, no. 6, pp. 2212-2223, 2015.

[24] S. Tang, Z. Ma, M. Xiao, and L. Hao, "Hybrid Transceiver Design for Beamspace MIMO-NOMA in Code-Domain for MmWave Communication Using Lens Antenna Array," IEEE Journal on Selected Areas in Communications, vol. 38, No. 9, pp.2118 - 2127, Sept. 2020.

[25] S. M. Tseng, Y. F. Chen, H. H. Fang,"Cross PHY/APP Layer User Grouping and Power Allocation for Uplink Multiantenna NOMA Video Communication Systems," IEEE Systems Journal, vol. 14, No. 3, pp. 3351 - 3359, Sept. 2020.

[26] C. Kai, Y. Wu, M. Peng, W. Huang,"Joint Uplink and Downlink Resource Allocation for NOMA-Enabled D2D Communications,'IEEE Wireless Communications Letters, vol. 10, no. 6, pp.1247 - 1251, June 2021.

[27] D. A. Sehrai, F. Muhammad, S. H. Kiani, Z. H. Abbas, M. Tufail, S. Kim, "Gain-Enhanced Metamaterial Based Antenna for 5G Communication Standards," CMC-Computers, Materials and Continua, vol.64, no.3, 2020, pp.1587-1599, doi:10.32604/cmc.2020.011057.

[28] D. Fan, F. Gao, G. Wang, Z. Zhong, A. Nallanathan,"Channel Estimation and Transmission Strategy for Hybrid MmWave NOMA Systems," IEEE Journal of Selected Topics in Signal Processing, vol.13, No. 3, pp. 584 596, June 2019.

[29] I. Granada, P. M. Crespo, J. G. Frías, "Rate Compatible Modulation for Non-Orthogonal Multiple Access," IEEE Access, vol. 8, pp. 224246 224259, 2020.

[30] L. Liu, B. Sun, X. Tan, D. H. K. Tsang, "Energy-Efficient Resource Allocation and Subchannel Assignment for NOMA-Enabled Multiaccess Edge Computing," IEEE Systems Journal 2021,DOI: 10.1109/JSYST.2021.3064919.

[31] A. H. Tacuri, J. Maldonado, M. Revollo, H. Chambi,"Bit Error Rate Analysis of NOMA-OFDM in 5G Systems With Non-Linear HPA With Memory," IEEE Access, vol. 9, pp.83709 - 83717, 2021.

[32] D. Song, W. Shin, J. Lee, H. V. Poor,"Sum-Throughput Maximization in NOMA-Based WPCN: A Cluster-Specific Beamforming Approach,'IEEE Internet of Things Journal, vol. 8, no. 13, pp.10543 - 10556, 2021

[33] F. Jia, C. Zhang, C. Jiang, M. Li, J. Ge, "Guaranteeing Positive Secrecy Rate for NOMA System Against Internal Eavesdropping," IEEE Communications Letters, vol. 25, no. 6, pp.1805 - 1809, June 2021.

[34] R. K. Ahiadormey, P. Anokye, H. S. Jo, C. Song, K. J. Lee, "Secrecy Outage Analysis in NOMA Power Line Communications," IEEE Communications Letters, vol. 25, no. 5, pp.1448 - 1452, May 2021. 
[35] X. Liu, Y. Liu, Y. Chen, and H. V. Poor, "RIS Enhanced Massive Nonorthogonal Multiple Access Networks: Deployment and Passive Beamforming Design," IEEE Journal on Selected Areas in Communications, vol.39, No.4, pp.1057 - 1071 April 2021.

[36] M. F. Sohail, C. Y. Leow, and S. Won, "Non-orthogonal multiple access for unmanned aerial vehicle assisted communication," IEEE Access, vol.6, pp. 22716-22727, 2018.

[37] H. Zhang, J. Zhang, K. Long, "Energy Efficiency Optimization for NOMA UAV Network with Imperfect CSI,” IEEE Journal on Selected Areas in Communications, vol. 38, No. 12, Dec. 2020.

[38] S. Khairy, P. Balaprakashy, L. X. Cai, Y. Cheng, "Constrained Deep Reinforcement Learning for Energy Sustainable Multi-UAV based Random Access IoT Networks with NOMA,'IEEE Journal on Selected Areas in Communications, volume. 39, No. 4, pp.1101 - 1115, April 2021

[39] L. Dai, B. Wang, M. Peng, and S. Chen,"Hybrid Precoding-Based Millimeter-Wave Massive MIMO-NOMA with Simultaneous Wireless Information and Power Transfer,'IEEE Journal on Selected Areas in Communications, vol. 37, No. 1, Jan. 2019

[40] F. Zhao, W. Hao, L. Shen, G. Sun, Y. Zhou, Y. Wang, "Secure Energy Efficiency Transmission for mmWave-NOMA System," IEEE Systems Journal, vol. 15, No. 2, pp. 2226 - 2229, June 2021.

[41] A. A. Badrudeen, C. Y. Leow, S. Won, "Sub-Connected Structure Hybrid Precoding for Millimeter-Wave NOMA Communications," IEEE Wireless Communications Letters, vol.10, no. 6, pp.1334 - 1338, June 2021

[42] "Updated high speed train scenario desciption," CMCC, China, Busan,Tech. Rep. R1-163867, Apr. 2016.

[43] "WF on evaluation assumptions for high speed train scenario: Macro + relay at 30GHz," ETRI, Korea, Nanjing, Tech. Rep. R1-165484, May 2016.

[44] S.Y. Lien, C.C Chien, H.L Tsai, Y.C Liang, D. I. Kim,"Configurable 3GPP Licensed Assisted Access to Unlicensed Spectrum," IEEE Wireless Communications, vol. 23, No. 6, pp.32 - 39, Dec. 2016.

[45] L. Zhu, Z. Xiao, X. G. Xia, D. O. Wu,"Millimeter-Wave Communications With Non-Orthogonal Multiple Access for B5G/6G,'IEEE Access, vol. 7, pp.116123 - 116132, 2019
[46] A. Roz'e, M. Crussi 'ere, M. H'elard, C. Langlais, "Comparison between a hybrid digital and analog beamforming system and a fully digital Massive MIMO system with adaptive beamsteering receivers in millimeterWave transmissions," International Symposium on Wireless Communication Systems (ISWCS), Sept. 2016.

[47] M. Abdelghany, A. A. Farid, U. Madhow, M. J. W. Rodwell, “Towards Alldigital mmWave Massive MIMO: Designing around Nonlinearities," 52nd Asilomar Conference on Signals, Systems, and Computers, Pacific Grove, CA, USA, 2019.

[48] X. Gao, L. Dai, Z. Chen, Z. Wang, and Z. Zhang, "Near-optimal beam selection for beamspace mmWave massive MIMO systems," IEEE Commun. Lett., vol. 20, no. 5, pp. 1054-1057, May 2016.

[49] J. Brady, N. Behdad and A. Sayeed, "Beamspace MIMO for millimeterwave communications: System architecture modeling analysis and measurement," IEEE Trans. Antennas Propag., vol. 61, no. 7, pp. 3814-3827, Jul.2013.

[50] S. Niknam, A. A. Nasir, H. Mehrpouyan and B. Natarajan, "A Multiband OFDMA Heterogeneous Network for Millimeter Wave 5G Wireless Applications," IEEE Access, vol. 4, pp.5640 - 5648, 2016.

[51] R. Melki, H.N Noura and A. Chehab, Physical layer security for NOMA limitations, issues, and recommendations. Ann. Telecommun. (2020), https://doi.org/10.1007/s12243-020-00819-7.

[52] Q. Li, P. Ren, Q. Du, D. Xu and Y. Xie, "Safeguarding NOMA Enhanced Cooperative D2D Communications via Friendly Jamming," 2019 IEEE 90th Vehicular Technology Conference (VTC2019-Fall), Honolulu, HI USA, 2019, pp. 1-5, doi: 10.1109/VTCFall.2019.8891295.

[53] Y. G. Li and G. L. Stuber, "Orthogonal frequency division multiplexing for wireless communications," Springer Science Business Media, 2006.

[54] H. G. Myung, J. Lim, and D. J. Goodman, "Single carrier FDMA for uplink wireless transmission," IEEE Vehicular Technology Magazine, vol 1, no. 3, pp. 30-38, 2006

[55] M. Zeng, A. Yadav, O. A. Dobre, G. I. Tsiropoulos, H. V. Poor, "Capacity Comparison Between MIMO-NOMA and MIMO-OMA With Multiple Users in a Cluster," IEEE Journal on Selected Areas in Communications, vol. 35 , No. 10, pp. 2413 - 2424, Oct. 2017. 